\title{
A comparison of BMI and Lipid Profile in patients with metabolic syndrome and Type 2 Diabetes Mellitus
}

\author{
Augusthy $A^{1}$, Sahu $S^{2}$, Ashok Kumar $J^{3}$, Jawalekar $S^{4}$, Marakala $V^{5}$, Thattil $A^{6}$ \\ ${ }^{1}$ Dr Asha Augusthy, Associate Professor, Department of Biochemistry, Sree Narayana Institute of Medical Sciences, \\ Ernakulam, Kerala, ${ }^{2}$ Dr. Suchanda Sahu, Associate Professor, Department of Biochemistry, All India Institute of Medical \\ Sciences, Sijua, Bhubaneshwar, Odisha, ${ }^{3}$ Dr. Ashok Kumar Jeppu, Associate Professor, Biochemistry unit, International \\ Medical School, Management and Science University, Shah Alam, Selangor, Malaysia, ${ }^{4}$ Dr. Seema Jawalekar, Professor, \\ Department of Biochemistry, Sree Narayana Institute of Medical Sciences, Ernakulam, Kerala, ${ }^{5}$ Dr.Vijaya Marakala, \\ Assistant Professor, Biochemistry unit, International Medical School, Management and Science University, Shah Alam, \\ Selangor, Malaysia, ${ }^{6}$ Dr. Anitha Maria Thomas Thattil, Assistant Professor, Department of Biochemistry, Yenepoya \\ Medical College, Deralakatte, Someshwar, Mangalore, Karnataka, India.
}

Address for Correspondence: Dr. Asha Augusthy, Associate Professor, Department of Biochemistry, Sree Narayana Institute of Medical Sciences, Ernakulam, Kerala. Email address:asha.augusty@gmail.com

\begin{abstract}
Introduction: Diabetes mellitus is a metabolic disease known by chronic hyperglycemia which results from defective insulin action and secretion. Metabolic syndrome consists of a constellation of metabolic abnormalities that confer increased risk of diabetes mellitus. The aim of the present study was to study BMI and lipid profile in patients with metabolic syndrome and type 2 diabetes mellitus. Materials and methods: 50 controls, 50 individuals with metabolic syndrome and 50 individuals with type 2 diabetes mellitus were selected by purposive sampling technique. BMI was calculated and serum levels of cholesterol, triglycerides, LDL, VLDL and HDL were estimated in controls and cases. Results: BMI, serum triglycerides, VLDL, cholesterol/HDL ratio were significantly increased $(\mathrm{p}<0.05)$ and serum HDL levels were significantly decreased $(\mathrm{p}<0.05)$ in metabolic syndrome and type 2 diabetes compared to controls. Conclusion: Our study concluded that there is significant dyslipidemia in patients with metabolic syndrome and type 2 diabetes mellitus.
\end{abstract}

Keywords: BMI, Diabetes mellitus, Lipid profile, Metabolic syndrome

\section{Introduction}

Diabetes mellitus is a metabolic disease known by chronic hyperglycemia which results from defective insulin action and secretion. World Health Organization projects that number of diabetics will exceed 350 million by 2030 . Various studies have documented liver disease as a major cause of mortality in patients with type 2 diabetes (T2DM). It is well known that liver plays an important role in maintenance of normal glucose levels during fasting as well as in the postprandial period .Metabolic syndrome (MetS) consists of a constellation of metabolic abnormalities that confer increased risk of diabetes mellitus.

Manuscript received: $6^{\text {th }}$ March 2017

Reviewed: $14^{\text {th }}$ March 2017

Author Corrected: $23^{\text {rd }}$ March 2017

Accepted for Publication: $31^{\text {st }}$ March 2017
The major features of the metabolic syndrome include central obesity, hypertriglyceridemia, low HDL cholesterol, hyperglycemia and hypertension [1].

Central feature of metabolic syndrome is insulin resistance. It results in hyperglycemia and hyperinsulinemia later leading to diabetes mellitus. It contributes to pathogenesis of various diseases like hypertension, atherosclerosis, coronary artery disease and organ dysfunctions [2].

Obesity is associated with a variety of cardiometabolic diseases, such as type 2 diabetes mellitus (T2DM), hypertension, hyperlipidemia, metabolic syndrome, and cardiovascular disease (CVD), all of which contribute 
to increased mortality. In addition, in a number of epidemiologic surveys, even in people deemed otherwise healthy and lacking any identifiable diseases or health risks, there is a higher risk for cardiometabolic dysfunction and mortality if they are overweight or obese [3].

Metabolic syndrome (MetS) and body mass index (BMI) are established independent risk factors in the development of diabetes. Obesity consists of heterogeneous phenotypes resulting from interplay between genetic and environmental factors. Increased BMI has been associated with metabolic and cardiovascular risk factors including diabetes, hypertension, dyslipidemia, but there is increasing evidence that sub-phenotypes of obesity exist that appear to deviate from the standard dose-response relationship between increased BMI and its adverse clinical outcomes [4].

Several studies have reported a strong association between excess weight and increased risk of death, placing the overweight group at a $40 \%$ higher and the obese group at up to $300 \%$ higher risk of death than individuals whose $\mathrm{BMI}$ is normal $(18.5 \leq \mathrm{BMI}<25)$.

Excess weight and physical inactivity are also associated with an increased risk of developing various diseases, particularly type 2 diabetes. Since excess weight is an important predictor of type $2 \mathrm{DM}$, the term "diabesity" was proposed by Astrup and Finer in 2000. Specifically, In comparison to women with normal BMI, overweight, obese class I and II (30 $\leq$ BMI < 39.99), and class III (BMI $\geq 40$ ) individuals face increased risks of developing type 2 DM with 7.6\%, $20.1 \%$ and $38.8 \%$ greater risk respectively [5].

Dyslipidemia contributes to the progression of atherosclerosis, the underlying pathology of CVD. Individuals with MetS or T2DM exhibit a characteristic pattern of abnormalities in serum lipid levels consisting of low levels of HDL-C and elevated levels of triglycerides (TG).

This dyslipidemia is also characterized by increased concentration of small, dense low-density lipoprotein cholesterol (LDL-C) particles [3]. Such lipid pattern is termed atherogenic dyslipidemia. Evidence from epidemiologic studies suggests that the co-occurrence of low levels of HDL-C and elevated levels of TG is a strong risk factor for CVD [6].
The aim of this study is to study BMI and lipid profile in patients with metabolic syndrome and type 2 diabetes.

\section{Materials and Methods}

Study design- The present study was conducted in the department of Biochemistry, Father muller's medical college after obtaining clearance from institutional ethics committee.

The study group consisted of 150 individuals selected by purposive sampling technique who had come to hospital for health check-up during a time period of two years. Informed written consent was obtained from all individual participants included in the study. This was a case-control study with a sample size of 150 patients.

Selection of subjects- 50 individuals with metabolic syndrome (all patients who fulfil criteria for metabolic syndrome, according to National cholesterol education program (NCEP): ATP III 2001 for metabolic syndrome [7]), 50 individuals with type 2 diabetes mellitus and 50 controls were selected.

Exclusion criteria- Smokers, alcoholics, patients with history of liver and renal impairment were excluded from the study.

Sample and data collection- For the selected patient's history was taken, physical examination was done and BMI was calculated as weight $(\mathrm{kg})$ divided by square of the height $\left(\mathrm{m}^{2}\right)$.

Serum cholesterol, LDL, HDL, Triglyceride levels were estimated. Serum levels of cholesterol, LDL were estimated using enzymatic colour test CHOD-PAP method and Triglycerides were estimated by enzymatic colour test GPO-PAP method. HDL was estimated by immune-inhibition enzymatic colour test. VLDL levels were calculated using Friedewald's equation.

All estimations were done on Olympus AU 400 autoanalyzer.

Statistical analysis- The data was analysed by ANOVA for multiple group comparisons and Pearson's correlation coefficient for relationship between variables. Statistical analyses were performed with the help of SPSS software. For all statistical analyses the $p$ value was considered to be significant when $\mathrm{p}<0.05$. 


\section{Results}

In our study, the BMI in metabolic syndrome, diabetes mellitus was more than controls. The BMI is $24.78 \pm 3.83$ in controls, $26.94 \pm 4.01$ in metabolic syndrome and $27.07 \pm 4.17$ in diabetes. Statistical analysis $(\mathrm{F}-5.177)$ shows that there is a significant increase in BMI in $(\mathrm{p}<0.001)$. metabolic syndrome and diabetes mellitus when compared to controls. We found that in patients with metabolic syndrome there is significant positive correlation of BMI with FBS. In the present study it was found that FBS, PPBS, serum triglycerides, serum VLDL and HDL ratio levels were significantly increased and serum HDL levels were significantly decreased in patients with metabolic syndrome and diabetes mellitus when compared to controls.In patients with metabolic syndrome there is significant positive correlation of BMI with FBS.

There is significant positive correlation of serum triglycerides with serum cholesterol, serum VLDL and HDL ratio in patients with metabolic syndrome. There is significant positive correlation of serum triglycerides with serum cholesterol, serum VLDL and HDL ratio in patients with diabetes mellitus.

Table-1: Distribution of cases and controls with respect to BMI [22]

\begin{tabular}{|c|c|c|c|}
\hline BMI & Controls & Metabolic syndrome & Diabetes mellitus \\
\hline Normal & 17 & 6 & 5 \\
$(18.5-22.9)$ & $(33.3 \%)$ & $(12.2 \%)$ & 13 \\
\hline At risk & 10 & 10 & $(26.5 \%)$ \\
$(23-24.9)$ & $(19.6 \%)$ & $(18.4 \%)$ & 19 \\
\hline Obese I & 20 & 28 & $(38.8 \%)$ \\
$(25-29.9)$ & $(39.2 \%)$ & $(57.1 \%)$ & 13 \\
\hline Obese II & 3 & 6 & $(24.5 \%)$ \\
$(>=30)$ & $(7.8 \%)$ & $(12.2 \%)$ & $\mathbf{5 0}$ \\
\hline Total & $\mathbf{5 0}$ & $\mathbf{5 0}$ & \\
\hline
\end{tabular}

Table-2: Comparison of FBS, PPBS, lipid profile between the 3 groups.

\begin{tabular}{|c|c|c|c|c|}
\hline & Control & Metabolic syndrome & Diabetes mellitus & p value \\
\hline FBS & $99.2 \pm 9.23$ & $111 \pm 8.49$ & $153.29 \pm 22.58$ & $<0.001$ \\
\hline PPBS & $105.82 \pm 39.41$ & $111.77 \pm 41.72$ & $176.4 \pm 52.28$ & $<0.001$ \\
\hline Cholesterol & $222.72 \pm 48.17$ & $223.16 \pm 48.47$ & $225.33 \pm 40.71$ & 0.955 \\
\hline HDL & $45.29 \pm 7.82$ & $38.22 \pm 5.82$ & $40.63 \pm 9.51$ & .001 \\
\hline LDL & $105 \pm 29.08$ & $107 \pm 28.47$ & $111.55 \pm 25.29$ & .519 \\
\hline VLDL & $27.54 \pm 19.59$ & $44.33 \pm 31.55$ & $46.91 \pm 30.65$ & .001 \\
\hline Triglycerides & $139.31 \pm 103.67$ & $217.98 \pm 143.25$ & $225 \pm 140.89$ & .002 \\
\hline Cholesterol/HDL Ratio & $5.18 \pm 0.9$ & $5.89 \pm 1.46$ & $5.39 \pm 1.71$ & .043 \\
\hline
\end{tabular}

Table-3: Correlation of triglycerides with FBS, PPBS and lipid profile in metabolic syndrome.

\begin{tabular}{|c|c|c|c|}
\hline & & Pearson correlation r value & p value \\
\hline Triglycerides & FBS & -0.718 & 0.598 \\
\hline & PPBS & -.105 & 0.580 \\
\hline & Cholesterol & .607 & 0.000 \\
\hline & HDL & -.257 & 0.075 \\
\hline & LDL & .145 & 0.325 \\
\hline & VLDL & .998 & 0.000 \\
\hline & $\begin{array}{c}\text { Cholesterol/ } \\
\text { HDL_ratio }\end{array}$ & .710 & 0.000 \\
\hline
\end{tabular}


Table-4: Correlation of triglycerides with FBS, PPBS and lipid profile in diabetes mellitus.

\begin{tabular}{|c|c|c|c|}
\hline & & Pearson correlation r value & p value \\
\hline Triglycerides & FBS & .154 & .294 \\
\hline & PPBS & .210 & .325 \\
\hline & Cholesterol & .387 & .007 \\
\hline & HDL & -.119 & .422 \\
\hline & LDL & .047 & .764 \\
\hline & VLDL & .971 & .000 \\
\hline & $\begin{array}{c}\text { Cholesterol/ } \\
\text { HDL_ratio }\end{array}$ & .258 & .091 \\
\hline
\end{tabular}

\section{BMI}

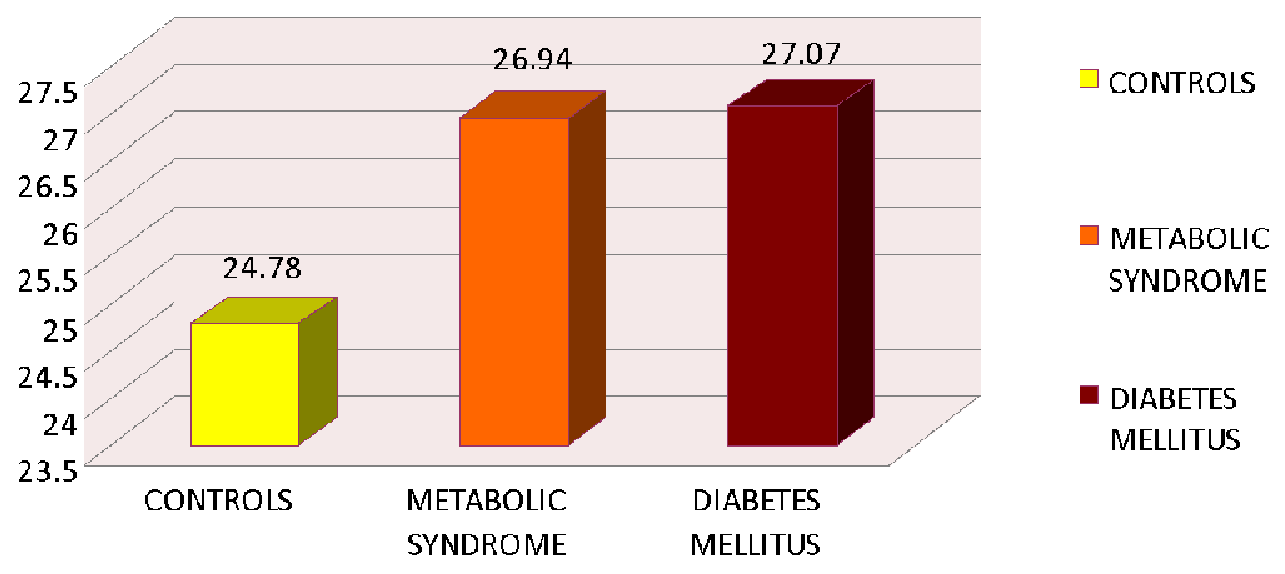

Fig-1: Comparison of BMI between controls, metabolic syndrome and diabetes mellitus $(p<0.001)$

\section{Discussion}

Diabetes mellitus is a metabolic disorder due to defect in the secretion of insulin and/or defect in the action of insulin characterized by hyperglycemia. Diabetes mellitus type 2 and metabolic syndrome are conditions associated with insulin resistance. Metabolic syndrome may be associated with dyslipidemia, hypertension, glucose intolerance, proinflammatory state, and a prothrombotic state [8].

BMI is significantly increased in patients with metabolic syndrome and type 2 diabetes compared to controls [4, 9-13]. Further it was noted that in patients with metabolic syndrome there is significant positive correlation of BMI with FBS. This is in accordance with study done by Vittal BG et al[14].

Individuals with MetS or T2DM exhibit a characteristic pattern of abnormalities in serum lipid levels consisting of low levels of HDL-C and elevated levels of TG.
Evidence from epidemiologic studies suggests that the co-occurrence of low levels of HDL-C and elevated levels of TG is a strong risk factor for CVD [6].

We found that the serum triglyceride levels were significantly increased $(\mathrm{p}<.001)$ in metabolic syndrome when compared to controls. The serum triglyceride levels in diabetics were further increased when compared to metabolic syndrome. Lipoprotein lipase (LPL) is the main enzyme responsible for clearing TGcontaining lipoproteins from the circulation. Impairment of LPL activity is associated with insulin resistance [6]. That is the reason for increase for in triglycerides levels in metabolic syndrome and type 2 diabetes mellitus. It was also found that there is significant positive correlation of serum triglycerides with serum cholesterol, serum VLDL and cholesterol/ HDL ratio in patients with metabolic syndrome and diabetes mellitus. 
In our study serum VLDL levels were significantly higher in metabolic syndrome and diabetes mellitus when compared to controls. This is in agreement with the work done by Songa RM and co-workers [15]. Serum VLDL is positively correlated with HDL ratio which is significant in patients with metabolic syndrome and diabetes mellitus. The HDL levels were significantly decreased in metabolic syndrome and diabetics when compared to controls.Hepatic lipase is the enzyme which clears HDL particles from the circulation. It shows increased activity in the presence of insulin resistance and as a result HDL-C levels decline. Low levels of HDL-C is an important risk factor for the development of CVD. The cardioprotective effects of HDL-C is due to its role in reverse cholesterol transport, its effects on endothelial cells, and its antioxidant activity [6].

Our studies are in accordance with previous studies which also found that there is hypertriglyceridemia and low HDL levels in metabolic syndrome and type 2 diabetes mellitus when compared to controls [6,15-20].

HDL ratio is significantly increased in patients with metabolic syndrome and diabetes mellitus when compared to controls. The TC/HDL ratio with raised values especially above 6 is a specific and sensitive index of cardiovascular risk and predictor of coronary heart disease [21-22].

Even Though total cholesterol and LDL cholesterol levels were increased in metabolic syndrome and diabetes mellitus, it was not found to be significant.

\section{Conclusion}

In the present study BMI and lipid profile were assessed in patients with metabolic syndrome and diabetes mellitus. It was found that serum triglycerides, VLDL levels were significantly increased in patients with metabolic syndrome and type 2 diabetes mellitus when compared with controls. Serum HDL levels were significantly decreased in patients with metabolic syndrome and diabetes mellitus when compared to controls. Our study concluded that there is significant dyslipidemia in patients with metabolic syndrome and type 2 diabetes mellitus which is a strong risk factor for CVD, so all type 2 diabetic patients should undergo lipid profile as a routine test.

Funding: Nil, Conflict of interest: None Permission of IRB: Yes
Acknowledgment: We thank all the individuals for willingly participating in this study. We acknowledge the support of the management of Father Muller's Medical College and Laboratory staff.

\section{References}

1. Augusthy A, Jeppu AK, Sahu S, Jawalekar S, Marakala V, Iqbal S. A study of liver functions in metabolic syndrome and Type 2 diabetes mellitus. Int $\mathbf{J}$ Med Res Rev 2016;4(4):470-475. doi: 10.17511/ijmrr. 2016.i04.01.

2. Kumar JA, Augusthy A. Oxidative stress in metabolic syndrome and Type 2 diabetes mellitus. Biomedicine. 2011;31(2):166-170.

3. Kuo JF, Hsieh YT, Mao IC, Lin SD, Tu ST, Hsieh MC. The Association Between Body Mass Index and All-Cause Mortality in Patients With Type 2 Diabetes Mellitus: A 5.5-Year Prospective Analysis. Medicine (Baltimore). 2015 Aug;94(34):e1398. doi: 10.1097/MD. 0000000000001398.

4. Hadaegh F, Bozorgmanesh M, Safarkhani M, Khalili D, Azizi F. "Predictability of body mass index for diabetes: affected by the presence of metabolic syndrome?". BMC Public Health. 2011 May 25;11:383. doi: 10.1186/1471-2458-11-383.

5. Gray N, Picone G, Sloan F, Yashkin A. Relation between BMI and diabetes mellitus and its complications among US older adults. South Med J. 2015 Jan; 108 (1):29-36. doi: 10.14423/SMJ.000000 0000000214.

6. Singh O, Gupta M, Khajuria V. Lipid profile and its relationship with blood glucose levels in Metabolic Syndrome. Natl J Physiol Pharm Pharmacol 2015;5 (2):134 - 137. doi: 0. 5455 / njppp. 2015. 5. 0511 20141.

7. Huang PL. A comprehensive definition for metabolic syndrome. Dis Model Mech. 2009 May-Jun;2(5-6): 231-7. doi: 10.1242/dmm.001180.

8. Jeppu AK, Augusthy A, Kumar KA. Plasma Glucose and Serum Ceruloplasmin in Metabolic Syndrome and Diabetes Mellitus Type 2. Recent Adv Biol Med. 2016; 2:15-19. 
9. Arnlov J, Sundström J, Ingelsson E, Lind L. Impact of BMI and the metabolic syndrome on the risk of diabetes in middle-aged men. Diabetes Care. 2011 Jan;34 (1): 61-5. doi: 10. 2337/dc10-0955. Epub 2010 Sep 17.

10. He Y,Jiang B, Wang J, Feng K,Chang Q, Zhu S, Fan L, Li X,Hu FB. BMI Versus the Metabolic Syndrome in Relation to Cardiovascular Risk in Elderly Chinese Individuals.Diabetes Care 2007 Aug; 30 (8): 2128-2134.

11. Arnlov J, Ingelsson E, Sundström J, Lind L. Impact of body mass index and the metabolic syndrome on the risk of cardiovascular disease and death in middle-aged men. Circulation. 2010 Jan 19;121(2):230-6. doi: 10.1161/CIRCULATIONAHA.109.887521. Epub 2009 Dec 28 .

12. Meigs JB, Wilson PW, Fox CS, Vasan RS, Nathan DM, Sullivan LM, D'Agostino RB. Body mass index, metabolic syndrome, and risk of type 2 diabetes or cardiovascular disease. J Clin Endocrinol Metab. 2006 Aug; 91 (8):2906-12. Epub 2006 May 30.

13. Ganz ML, Wintfeld N, Li Q, Alas V, Langer J, Hammer M. The association of body mass index with the risk of type 2 diabetes: a case-control study nested in an electronic health records system in the United States. Diabetol Metab Syndr. 2014 Apr 3;6(1):50. doi: 10.1186/1758-5996-6-50.

14. Vittal BG,Praveen G, Deepak P. A study of body mass index in healthy individuals and its relationship with fasting blood sugar. Journal of Clinical and Diagnostic Research [serial online] 2010 December [cited: 2017 Feb 16 ]; 4:3421-3424.

15. Songa RM, Viswabarathi N, KS. Comparative study of lipid profile in obese type 2 diabetes mellitus and obese non diabetes. IOSR Journal of Dental and Medical Sciences Nov. 2015;14(11) : 87-91.doi: 10. 9790/ 0853-141118791.

16. Krauss RM. Lipids and lipoproteins in patients with type 2 diabetes. Diabetes Care. 2004 Jun; 27 (6): 1496-504.

17. Brunzell JD, Ayyobi AF. Dyslipidemia in the metabolic syndrome and type 2 diabetes mellitus. Am J Med. 2003 Dec 8;115 Suppl 8A:24S-28S.

18. Alshehri AM. Metabolic syndrome and cardiovascular risk. J Family Community Med. 2010 May; 17(2):73-8. doi: 10.4103/1319-1683.71987.

19. Nsiah K, Shang VO, Boateng KA, Mensah FO. Prevalence of metabolic syndrome in type 2 diabetes mellitus patients. Int J Appl Basic Med Res. 2015 MayAug; 5 (2):133-8. doi: 10.4103/2229-516X.157170.

20. Ugle SS, Bikkad MD. Altered triglycerides and HDL-c are better marker for coronary heart disease in NIDDM. Int J Res Med Sci 2016;4:461-4. doi:http://dx.doi. org/ 10. 18203 /2320-6012. Ijrms 20160296.

21. Tangvarasittichai S, Poonsub P, Tangvarasittichai O. Association of serum lipoprotein ratios with insulin resistance in type 2 diabetes mellitus. Indian J Med Res. 2010 May;131:641-8.

22. Garg N, Agrawal YB, Gupta S.A study of lipid profile levels in diabetics and nondiabetics taking TC/HDL ratio and LDL/HDL ratio into consideration. JIACM 2014; 15(3-4): 192-5.

23. WHO /IASO/ IOTF. The Asia-Pacific perspective : redefining obesity and its treatment. Health Communications Australia:Melbourne, 2000.

\section{How to cite this article?}

Augusthy A, Sahu S, Ashok Kumar J, Jawalekar S, Marakala V, Thattil A. A comparison of BMI and Lipid Profile in patients with metabolic syndrome and Type 2 Diabetes Mellitus. Int J Med Res Rev 2017;5(03):357-362 doi:10.17511/ijmrr. 2017.i03.23. 